\title{
The Importance of Clinical Decision-Making in Surgical Planning for Non-Small Cell Lung Cancer
}

\author{
Hollis Johanson, MD, and Ikenna Okereke, MD \\ Department of Surgery, Henry Ford Health System, Detroit, MI
}

Lung cancer is the leading cancer killer in the United States and the world. Surgical resection remains an important part of the treatment for non-small cell lung cancer (NSCLC). Achieving negative surgical margins is a critical factor in prognosis, and patients who have positive margins experience worse survival.

The study by van Joolingen and colleagues ${ }^{1}$ analyzed the ability of a nomogram they created to predict an R1 or $\mathrm{R} 2$ resection in locally advanced NSCLC. This nomogram, called the Rasing score, uses characteristics of the tumor and planned surgical approach to assign a score. Tumor histology, clinical $\mathrm{T}$ stage, clinical $\mathrm{N}$ stage, extent of planned surgical resection (lobectomy, sub-lobar or wedge resection, pneumonectomy, sleeve resection), and thoracoscopic versus open surgery are used as determinants of the Rasing score. ${ }^{2}$

Patients are further divided into low-risk (Rasing score 0-2), medium-risk (Rasing score 2-4), and high-risk (Rasing score $\geq 4$ ) cohorts for $\mathrm{R} 1 / \mathrm{R} 2$ resection. In the authors' previous study, the probabilities of these groups having a $\mathrm{R} 1 / \mathrm{R} 2$ resection were respectively $3.3 \%, 8.4 \%$, and $19.8 \%$. The current study used this prediction model to analyze whether definitive chemoradiation for these patients would resulted in improved overall survival for the high-risk group with a Rasing score higher than 4.

As demonstrated in the authors' report, the majority of the patients (78.2\%) with a Rasing score lower than 4 experienced an $\mathrm{R} 0$ resection. In the analysis of the patients

(C) Society of Surgical Oncology 2021

First Received: 11 October 2021

Accepted: 20 October 2021;

Published Online: 8 November 2021

I. Okereke, MD

e-mail: iokerek1@hfhs.org in the high-risk group (Rasing score $>4$ ), those with an R0 resection had a median survival of 45.6 months and experienced better survival than the patients who underwent definitive chemoradiation treatment. The patients with positive resection margins had a poorer median survival than their matched chemoradiation cohort.

This article demonstrates the limitation of relying on a nomogram alone to predict the ability to achieve a complete resection. When the resectability of a tumor is evaluated, multiple factors are used. The National Comprehensive Cancer Network (NCCN) publishes guidelines for addressing cancer treatment developed by agreement of leaders in the field of surgical and medical oncology. ${ }^{3}$ Clinical stage, lymph node status, patient functionality, and staging workup all are characteristics that determine suitability for surgery.

The Rasing score accounts for the planned surgical resection, but it fails to capture the nuances routinely used in the selection of patients for surgery. In addition, imaging characteristics such as relationship to the great vessels and other intrathoracic structures are not captured in the Rasing score nomogram.

Not only tumor characteristics are important in predicting an R0 resection, but patient functional status also needs to be addressed in the selection of the best treatment approach. Pulmonary function tests, patient frailty, and exercise tolerance all are important points to consider in determining eligibility for surgery. These points are especially vital for patients who may require an extended resection, such as a bi-lobectomy, to achieve negative margins. For patients who do not seem able to tolerate the necessary lung resection, neoadjuvant or definitive chemoradiation may be a better option.

Additionally, the patient's support system is an important factor to consider during the preoperative planning phase. For patients who have limited social support, 
unsheltered housing, or continued tobacco/substance use, addressing these issues before surgery will give them a better chance to tolerate surgical resection. For these reasons, the treatment approach should be discussed in a multidisciplinary setting with consideration of patient factors and tumor characteristics.

In the management of lung cancer patients, the best treatment approach is based on multiple factors. Tumor location, size, presence of nodal metastases, and predicted extent of resection guide the surgeon to choose the best suitability for surgery. Furthermore, patient frailty and the patient's support system also will guide the treatment plan.

The authors should be applauded for their attempt to create a predictive model to guide decision-making. But this model should be used with other factors to determine the best treatment plan for the patient.

DISCLOSURE There are no conflicts of interest.

\section{REFERENCES}

1. van Joolingen HW, Rasing M, Peters M, et al. Non-Small Cell Lung Cancer Patients with a High Predicted Risk of Irradical Resection: Can Chemoradiotherapy Offer Similar Survival? Ann Surg Oncol. 2021. https://doi.org/10.1245/s10434-021-10982-3

2. Rasing MJA, Peters M, Moreno AC, et al. Predicting Incomplete Resection in Non-small Cell Lung Cancer Preoperatively: A Validated Nomogram. Ann Thorac Surg. 2021;111:1052-8.

3. Ettinger DS, Akerley W, Bepler G, et al. Non-Small Cell Lung Cancer. J Natl Compr Cancer Network. 2010;8:740-801.

Publisher's Note Springer Nature remains neutral with regard to jurisdictional claims in published maps and institutional affiliations. 